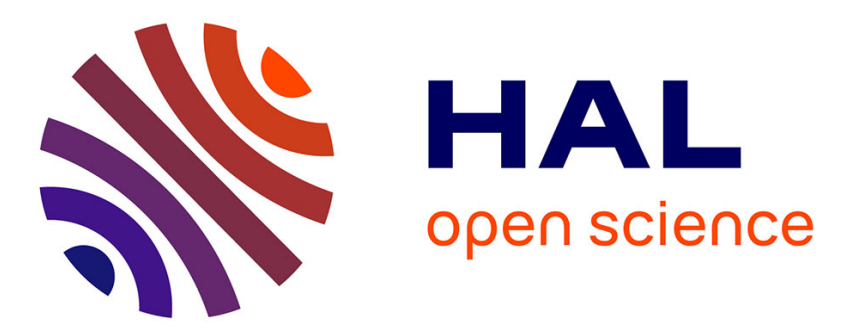

\title{
Thibaut Muzergues, La quadrature des classes. Comment de nouvelles classes sociales bouleversent les systèmes de partis en Occident \\ Camille Couvry
}

\section{- To cite this version: \\ Camille Couvry. Thibaut Muzergues, La quadrature des classes. Comment de nouvelles classes sociales bouleversent les systèmes de partis en Occident. 2018. hal-02184934}

HAL Id: hal-02184934

https://hal-normandie-univ.archives-ouvertes.fr/hal-02184934

Submitted on 16 Jul 2019

HAL is a multi-disciplinary open access archive for the deposit and dissemination of scientific research documents, whether they are published or not. The documents may come from teaching and research institutions in France or abroad, or from public or private research centers.
L'archive ouverte pluridisciplinaire HAL, est destinée au dépôt et à la diffusion de documents scientifiques de niveau recherche, publiés ou non, émanant des établissements d'enseignement et de recherche français ou étrangers, des laboratoires publics ou privés. 


\section{Thibaut Muzergues, La quadrature des classes.}

Comment de nouvelles classes sociales bouleversent les systèmes de partis en Occident

\section{Camille Couvry}

\section{(2) OpenEdition \\ Journals}

Édition électronique

URL : http://journals.openedition.org/lectures/25174

ISSN : 2116-5289

\section{Éditeur}

Centre Max Weber

Ce document vous est offert par Université de Rouen - Bibliothèque Universitaire

\section{UDUNIVERSITÉ}

Référence électronique

Camille Couvry, «Thibaut Muzergues, La quadrature des classes. Comment de nouvelles classes sociales bouleversent les systèmes de partis en Occident », Lectures [En ligne], Les comptes rendus, 2018, mis en ligne le 28 juin 2018, consulté le 16 juillet 2019. URL : http://journals.openedition.org/lectures/25174

Ce document a été généré automatiquement le 16 juillet 2019.

(c) Lectures - Toute reproduction interdite sans autorisation explicite de la rédaction / Any replication is submitted to the authorization of the editors 


\title{
Thibaut Muzergues, La quadrature des classes. Comment de nouvelles classes sociales bouleversent les systèmes de partis en Occident
}

\author{
Camille Couvry
}

1 En France, les résultats du premier tour des élections présidentielles de 2017 ont été serrés entre les quatre premiers candidats et ont montré que le clivage binaire entre gauche et droite n'était plus aussi saillant que par le passé. Considérant que cette situation relève d'une transformation profonde des systèmes politiques en Occident, Thibault Muzergues défend la thèse selon laquelle quatre catégories différentes d'électeurs sont actuellement influentes et redéfinissent le système politique en un jeu quadripartite. Les quatre «classes sociales» identifiées par l'auteur ne sont pas nécessairement antagonistes et se distinguent en ce sens d'une acception marxienne du terme. Quel est leur profil sociologique ? Comment ces nouvelles classes redéfinissent-elle l'offre politique et interagissent-elles par le jeu des coalitions ? Portant un regard croisé sur les situations sociologiques et politiques dans différents pays de l'Union européenne et d'Amérique du Nord, cet ouvrage propose de faire la lumière sur les reconfigurations de l'électorat et des coalitions dans le monde occidental tout en identifiant quelques stratégies gagnantes et scénarios possibles pour les prochaines élections.

2 Tout d'abord, la classe créative ${ }^{1}$ regroupe les personnes diplômées qui exercent des métiers nécessitant de créer quelque chose. Les membres de cette classe tendent à indifférencier le temps de travail et la vie personnelle, recherchent les espaces denses, habitent les grands centres urbains et sont favorables à la diversité. Cette classe constitue la base électorale qui a soutenu Macron en France, Obama aux États-Unis ou encore Trudeau au Canada.

3 Très différente de la classe créative en termes de valeurs et de mode de vie, la classe moyenne provinciale est quant à elle installée dans les banlieues pavillonnaires ou dans les villes moyennes. Ses choix de vie s'orientent plutôt vers l'habitat en maison 
individuelle avec jardin et sur l'usage de la voiture. Le travail reste pour elle une valeur centrale qu'elle distingue nettement de la vie personnelle et des loisirs. Cette classe défend le système en place mais tend à s'élever contre ceux qu'elle perçoit comme des "profiteurs du système» (p. 58). En France, les membres de cette classe moyenne provinciale ont soutenu Fillon au premier tour et se sont éventuellement reportés sur Macron au second tour.

4 La troisième classe regroupe la "nouvelle minorité $»^{2}$ ouvrière blanche. Ses membres n'ont pas le sentiment d'être les plus défavorisés mais ont vu leur pouvoir politique diminuer et leurs conditions de vie stagner, voire se dégrader. Délaissé par la gauche, cet électorat soutient désormais dans plusieurs pays des partis populistes de droite (le Front national en France, Trump aux États-Unis, l'Alternative pour l'Allemagne outre Rhin, le Parti pour l'indépendance du Royaume-Uni en Grande-Bretagne). Ces partis ont intégré à leur rhétorique protectionniste, marquée par le refus de l'immigration, les préoccupations de cette classe en matière de protections sociales « avec un accent mis sur l'ancien modèle social national» (p. 72).

5 Enfin, la classe des «millenials » est celle des jeunes nés entre 1982 et 1996 qui sont éduqués et diplômés. Ils se sont formés à l'université durant plusieurs années mais accèdent à des emplois moins qualifiés et moins valorisés que les positions auxquelles ils aspirent, ce qui entraîne chez eux une désillusion et une attirance pour des partis populistes de gauche. Ce sont ces jeunes qui se sont engagés dans les mouvements sociaux comme « Occupy Wall Street » en 2010-2012 aux États-Unis ou « Nuit Debout » en 2016 en France. Ils ont récemment donné leur voix à des candidats tels que Sanders aux ÉtatsUnis, Mélenchon en France et moins récemment, Iglesias en Espagne et Tsípras en Grèce.

6 Si ces quatre classes permettent « de redonner un sens à un monde politique décomposé ou plutôt en instance de recomposition » (p. 86), elles ne sont pas des boîtes hermétiques et un individu est susceptible de s'identifier à plusieurs d'entre elles au cours de son cycle de vie. Par ailleurs, ces classes interagissent et peuvent créer des coalitions.

7 L'une des coalitions gagnantes - entre la classe créative et les millenials - a conduit aux victoires d'Obama en 2008 et 2012 et de Hollande en 2012. Une stratégie pour un candidat ou un parti consiste alors à étendre sa base électorale à une autre classe que celle dont il a le soutien au départ. Trump a été élu aux États-Unis en étendant sa base électorale à une partie de la classe moyenne provinciale alors qu'il avait déjà le soutien de la minorité ouvrière, tandis qu'en France, Macron a également obtenu le soutien d'une partie de la classe moyenne provinciale après l'affaire de Pénélope Fillon. En revanche, les partis sont parfois incapables de réorienter leur ligne idéologique en fonction de la nouvelle vision de leur base électorale, ce qui peut aboutir à des pertes brutales de leur influence, comme en Autriche où les deux partis historiques - les Sociaux-démocrates autrichiens (SPÖ) et le Parti populaire autrichien (ÖVP) - se sont fait éliminer au premier tour des présidentielles en 2016.

8 Pour l'auteur, il importe de tenir compte des rapports de force entre les classes, des clivages qui les traversent et de leur situation socio-économique pour comprendre les positionnements politiques récents. Par exemple, deux classes se distinguent en Europe centrale et orientale : la minorité ouvrière (majoritaire) et la classe moyenne provinciale. Pour des raisons économiques et démographiques liées à l'histoire de ces pays anciennement intégrés au bloc communiste, les créatifs et les millenials y sont moins nombreux que dans d'autres parties de l'Europe. Ces deux classes ne parviennent donc pas à exercer une forte influence sur la scène politique. Les partis au pouvoir dans 
certains pays comme la Hongrie adoptent une ligne idéologique (la défense des traditions, le développement du nationalisme et le rejet du libéralisme qu'incarne la classe créative) qui coïncide en réalité avec les visions des deux classes majoritaires. En revanche, certains pays comme l'Ukraine et la Roumanie ont vu la classe des créatifs monter peu à peu en puissance, ce qui a pu conduire à des transformations du paysage politique. Par exemple, en Roumanie, Klaus Iohannis a défendu la lutte contre la corruption et a ainsi obtenu le soutien des créatifs des centres urbains aux élections présidentielles de 2014.

Thibault Muzergues propose une théorie audacieuse répondant à l'objectif de rendre compte de la transformation des électorats, qu'il observe dans le monde occidental d'aujourd'hui à partir de l'identification de quatre classes influentes. Quatre remarques peuvent toutefois être faites. L'analyse tend à homogénéiser les catégories identifiées et à les transposer d'un contexte à un autre sans spécifier les conditions sociohistoriques et culturelles de chaque pays ni présenter les caractéristiques de leur champ politique (les éléments structurels, les modes de scrutin, etc.), ce qui rend les comparaisons parfois inadéquates. L'auteur étaye ses arguments en s'appuyant sur un volume diversifié de documents issus de la presse, d'instituts de statistique et de recherche, d'instituts de sondage et mobilise de nombreuses références scientifiques éclairantes pour situer ses influences théoriques. En revanche, son ancrage scientifique et méthodologique mériterait d'être davantage explicité. On regrette aussi qu'aucune référence ne soit faite aux débats suscités par la thèse de la "classe créative » en ce qui concerne tant les aspects méthodologiques (l'indice gay par exemples) que conceptuels (le flou de la définition et son apparente homogénéité4). Une prise en compte de ces critiques aurait peut-être permis de mieux expliquer certains mouvements que l'auteur identifie, concernant par exemple le (dé)classement des professeurs de secondaire ou des médecins généralistes et le glissement des "créatifs déclassés» (p.149) vers la catégorie des millenials. Enfin, si seules quatre classes apparaissent assez homogènes et influentes pour être identifiées comme telles, il est dommage que les populations ethniques soient absentes de l'analyse hormis en tant que sujets de cristallisation des divergences de visions du monde entre les populations blanches. L'ouvrage n'en reste pas moins très riche d'enseignements pour comprendre la sociologie électorale actuelle et les réalignements politiques en train de se dessiner dans les pays occidentaux.

\section{NOTES}

1. Florida Richard, The Rise of the Creative Class (revisited), New York, Basic Books, 2012.

2. Justin Gest, The New Minority. White Working Class Politics in an Age of Immigration and Inequality, Oxford University Press, 2016.

3. Un aperçu synthétique des critiques en français est disponible dans les notes de lecture suivantes: Chantelot Sébastien, "La thèse de la "classe créative": entre limites et développements ", Géographie, économie, société, vol. 11, n 4, 2009, p. 315-334, disponible en ligne : https://www.cairn.info/revue-geographie-economie-societe-2009-4-page-315.htm ; Sébastien Darchen et Diane-Gabrielle Tremblay «La thèse de la "classe créative" : son indice sur l'analyse 
des facteurs d'attraction et de la compétitivité urbaine ", Interventions économiques, $\mathrm{n}^{\circ} 37$ ? 2008, en ligne : https://journals.openedition.org/interventionseconomiques/503.

4. Jean-Pierre Durand, «Richard Florida, The Rise of the Creative Class, Revisited, New York, Basic Books, 2012, 484 p.», La nouvelle revue $d u$ travail, $\mathrm{n}^{\circ} 9,2016$, en ligne: http:// journals.openedition.org/nrt/2971.

\section{AUTEUR}

\section{CAMILLE COUVRY}

Chercheuse au laboratoire DySoLab, j'étudie les pratiques esthétiques articulées à la position sociale, au genre et à l'âge, notamment dans les concours de beauté, et je co-organise le séminaire itinérant et international « Corps et beauté ». 